\begin{tabular}{|c|l|}
\hline Title & Evidence for a secondary bow in Newton's zero-order nuclear rainbow \\
\hline Author(s) & Ohkubo, S.; Hirabay ashi, Y. \\
\hline Citation & $\begin{array}{l}\text { Physical Review C, 89(5), 051601 } \\
\text { https://doi.org/10.1103/PhysRevC.89.051601 }\end{array}$ \\
\hline Issue Date & 201405-08 \\
\hline Doc URL & http://hdl.handle.net/2115/56863 \\
\hline Rights & @2014 A merican Physical Society \\
\hline Type & article \\
\hline File Information & PhysRevC.89.051601.pdf \\
\hline
\end{tabular}

Instructions for use 


\title{
Evidence for a secondary bow in Newton's zero-order nuclear rainbow
}

\author{
S. Ohkubo ${ }^{1,2}$ and Y. Hirabayashi ${ }^{3}$ \\ ${ }^{1}$ Research Center for Nuclear Physics, Osaka University, Ibaraki, Osaka 567-0047, Japan \\ ${ }^{2}$ Department of Applied Science, Kochi Women's University, 5-15 Eikokuji-cho, Kochi 780-8515, Japan \\ ${ }^{3}$ Information Initiative Center, Hokkaido University, Sapporo 060-0811, Japan \\ (Received 12 December 2013; revised manuscript received 17 April 2014; published 8 May 2014)
}

\begin{abstract}
Rainbows are generally considered to be caused by static refraction and reflection. A primary and a secondary rainbow appear due to refraction and internal reflection in a raindrop as explained by Newton. The quantum nuclear rainbow, which is generated by refraction in the nucleus droplet, only has a "primary" rainbow. Here we show for the first time evidence for the existence of a secondary nuclear rainbow generated dynamically by coupling to an excited state without internal reflection. This has been demonstrated for experimental ${ }^{16} \mathrm{O}+{ }^{12} \mathrm{C}$ scattering using the coupled channel method with an extended double folding potential derived from microscopic realistic wave functions for ${ }^{12} \mathrm{C}$ and ${ }^{16} \mathrm{O}$.
\end{abstract}

DOI: 10.1103/PhysRevC.89.051601

PACS number(s): 24.10.Eq, 24.10.Ht, 25.55.Ci

Newton, who explained the mechanism of the rainbow by refraction and reflection [1-3], believed in the existence of the zero-order rainbow without internal reflection in a droplet. Newton's zero-order rainbow [4] was realized in quantum systems when Goldberg et al. [5] observed a nuclear rainbow in high-energy $\alpha$-particle scattering. The quantum nuclear rainbow is generated by the nuclear potential, which acts as a Luneburg lens [6], where refraction is the only active mechanism and a "primary" rainbow can only exist because there are no higher-order terms like in Nussenzveig's expansion for the meteorological rainbow [7]. We report here evidence for the unexpected existence of a secondary bow in the nuclear rainbow.

The nuclear rainbow is very important in determining the interaction potential family up to the internal region without discrete ambiguity [5] and has been studied extensively [8,9]. The nuclear rainbow is also powerful for studies of nuclear cluster structure in the bound and unbound energy regions. In fact, the global nuclear potential, which describes nuclear rainbow scattering for typical $\alpha+{ }^{16} \mathrm{O}, \alpha+{ }^{40} \mathrm{Ca}$, and ${ }^{16} \mathrm{O}+$ ${ }^{16} \mathrm{O}$ systems, can reproduce scattering over a wide range of incident energies and cluster structures of ${ }^{20} \mathrm{Ne},{ }^{44} \mathrm{Ti}$, and ${ }^{32} \mathrm{~S}$ in the bound and unbound energy regions, respectively, in a unified way [10-12]. In contrast to the ${ }^{16} \mathrm{O}+{ }^{16} \mathrm{O}$ system, the Airy structure of the nuclear rainbow for the asymmetric ${ }^{16} \mathrm{O}+{ }^{12} \mathrm{C}$ system, for which refraction is very strong, is clearly observed in experimental angular distributions without being obscured by symmetrization. For this system, a global potential that reproduces the energy evolution of the Airy minimum in the angular distributions over a wide range of incident energies at $E_{L}=62 \sim 1503 \mathrm{MeV}$ [13-17] also successfully explains the cluster structure and molecular resonances with the ${ }^{16} \mathrm{O}+{ }^{12} \mathrm{C}$ configuration in ${ }^{28} \mathrm{Si}$ in a unified way [18]. However, the global potential was confronted with a serious difficulty when a new measurement of the angular distribution in ${ }^{16} \mathrm{O}+{ }^{12} \mathrm{C}$ scattering at $E_{L}=281 \mathrm{MeV}$ up to $\theta \approx 100^{\circ}$ showed that an Airy minimum of the nuclear rainbow appears at the much larger angle $\theta \approx 70^{\circ}[19]$ than expected in the global potential $\left(\theta \approx 45^{\circ}\right)$. It has been impossible to model a first-order Airy minimum $A 1$ at this large angle with the established global potential [19]. Thus, the reason why the global potential fails to reproduce the Airy minimum of the nuclear rainbow at $E_{L}=281 \mathrm{MeV}$ has been a mystery and raised a serious question of the common belief that nuclear rainbow scattering can determine the potential uniquely.

The purpose of this paper is to present for the first time evidence for the existence of a secondary nuclear rainbow in the ${ }^{16} \mathrm{O}+{ }^{12} \mathrm{C}$ scattering generated dynamically by a quantum effect without internal reflections of classical concept, which is completely different from the Newton's meteorological secondary rainbow. We show that the Airy minimum at around $\theta \approx 70^{\circ}, A 1^{(S)}$, is not a first-order Airy minimum, $A 1^{(P)}$, of the well-known ("primary") nuclear rainbow due to the nuclear potential but a first-order Airy minimum of a secondary bow of the nuclear rainbow. The difficulty of the global potential is solved by the discovery of the secondary nuclear rainbow.

We study rainbow scattering for ${ }^{16} \mathrm{O}+{ }^{12} \mathrm{C}$ with an extended double folding (EDF) model that describes all the diagonal and off-diagonal coupling potentials derived from the microscopic realistic wave functions for ${ }^{12} \mathrm{C}$ and ${ }^{16} \mathrm{O}$ using a density-dependent nucleon-nucleon force. The diagonal and coupling potentials for the ${ }^{16} \mathrm{O}+{ }^{12} \mathrm{C}$ system are calculated using the EDF model without introducing a normalization factor:

$$
\begin{aligned}
V_{i j, k l}(\mathbf{R})= & \int \rho_{i j}^{\left({ }^{16} \mathrm{O}\right)}\left(\mathbf{r}_{1}\right) \rho_{k l}^{\left({ }^{12} \mathrm{C}\right)}\left(\mathbf{r}_{2}\right) \\
& \times v_{N N}\left(E, \rho, \mathbf{r}_{1}+\mathbf{R}-\mathbf{r}_{2}\right) d \mathbf{r}_{1} d \mathbf{r}_{2},
\end{aligned}
$$

where $\rho_{i j}^{\left({ }^{16} \mathrm{O}\right)}(\mathbf{r})$ is the diagonal $(i=j)$ or transition $(i \neq j)$ nucleon density of ${ }^{16} \mathrm{O}$ taken from the microscopic $\alpha+{ }^{12} \mathrm{C}$ cluster model wave functions calculated in the orthogonality condition model (OCM) in Ref. [20], which uses a realistic size parameter both for the $\alpha$ particle and ${ }^{12} \mathrm{C}$ and is an extended version of Ref. [21], which reproduces almost all the energy levels well up to $E_{x} \approx 13 \mathrm{MeV}$ and the electric transition probabilities in ${ }^{16} \mathrm{O}$. The wave functions have been successfully used for the systematic analysis of elastic and inelastic $\alpha+{ }^{16} \mathrm{O}$ scattering over a wide range of incident energies and the $\alpha$ cluster structure study of ${ }^{20} \mathrm{Ne}[22] . \rho_{k l}^{\left({ }^{12} \mathrm{C}\right)}(\mathbf{r})$ represents the diagonal $(k=l)$ or transition $(k \neq l)$ nucleon 
density of ${ }^{12} \mathrm{C}$, which is calculated using the microscopic three- $\alpha$-cluster model in the resonating group method [23]. This model reproduces the $\alpha$ cluster, $\alpha$ condensate, and shelllike structures of ${ }^{12} \mathrm{C}$ well and the wave functions have been checked for many experimental data, including charge form factors and electric transition probabilities. For the effective interaction $v_{\mathrm{NN}}$ we use the DDM3Y-FR interaction [24], which takes into account the finite-range nucleon exchange effect. An imaginary potential with a Woods-Saxon volume-type form factor is introduced phenomenologically to take into account the effect of absorption due to other channels.

First we show that the puzzling nuclear rainbow for the ${ }^{16} \mathrm{O}+{ }^{12} \mathrm{C}$ system at $E_{L}=281 \mathrm{MeV}$ cannot be explained by the traditional view that a rainbow is generated by a strong attractive nuclear potential. In Fig. 1, the angular distributions of elastic ${ }^{16} \mathrm{O}+{ }^{12} \mathrm{C}$ scattering calculated using the conventional single-channel double-folding (DF) potential without channel couplings (blue solid lines) and the refractive farside components (dashed lines) are compared with the experimental

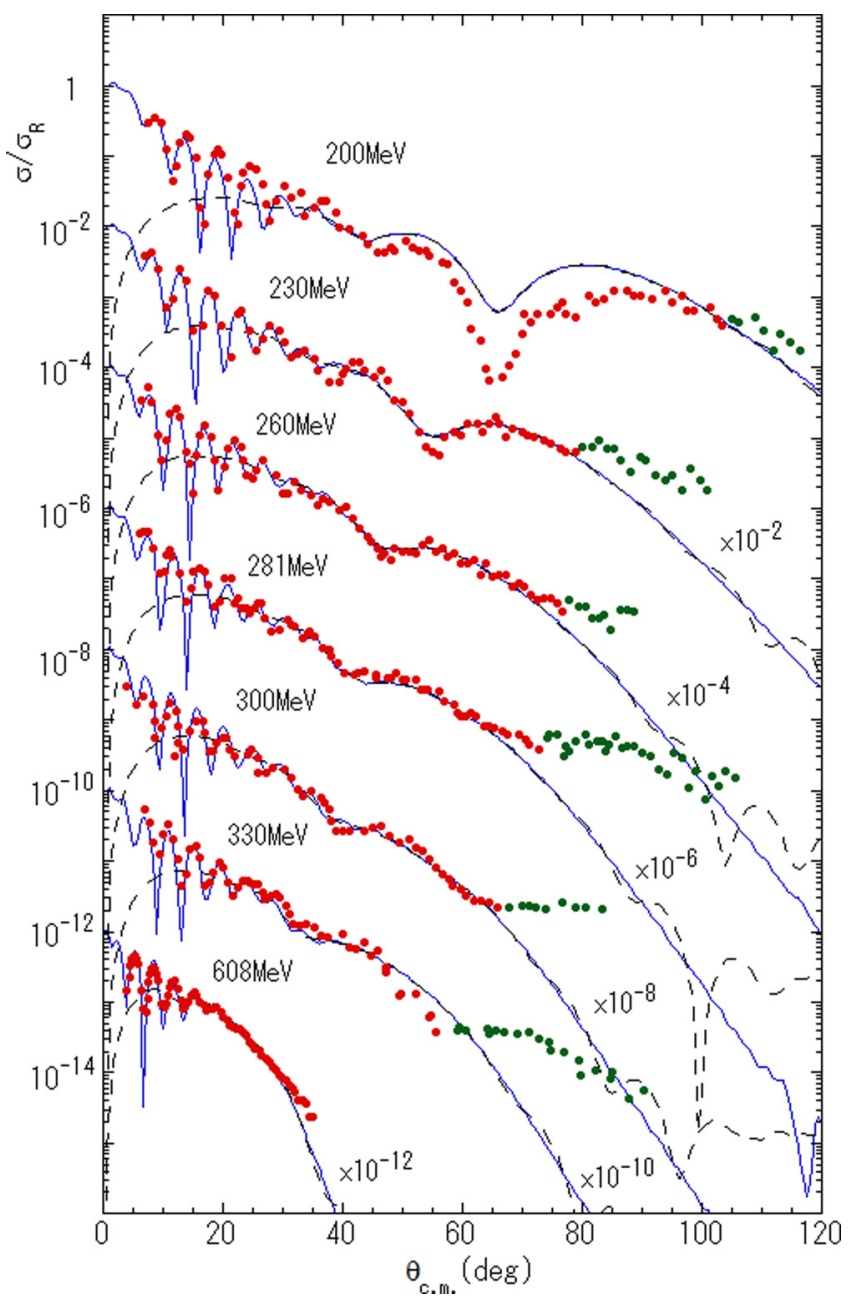

FIG. 1. (Color online) Comparison of the conventional singlechannel DF potential calculations (blue solid line) with experimental angular distributions (red and green points) in ${ }^{16} \mathrm{O}+{ }^{12} \mathrm{C}$ rainbow scattering [15,25-27]. The long dashed line displays the calculated farside components. data (points) at $E_{L}=200 \sim 260 \mathrm{MeV}$ [15], $281 \mathrm{MeV}$ [19], $300 \mathrm{MeV}$ [25], $330 \mathrm{MeV}$ [26], and $608 \mathrm{MeV}$ [27]. For the imaginary potential, the strength parameter around $20 \mathrm{MeV}$ was found to fit the data while the radius parameter and the diffuseness parameter were fixed at around 5.6 and $0.65 \mathrm{fm}$, respectively. The potential parameters used and the values of the volume integral per nucleon pair of the DF potential, $J_{V}$, are given in Table I. We found that the DF potential works well without introducing a normalization factor, values of the volume integral per nucleon pair are consistent with those used in other DF optical model calculations [14,15,17,25] and the DF potential used belongs to the same global potential family found in the $E_{L}=62 \sim 124 \mathrm{MeV}$ [14] and $E_{L}=$ $132 \sim 1503 \mathrm{MeV}$ regions [15,17]. We see that the angular distributions are dominated by the refractive farside scattering. In Fig. 1, the discrepancies are clear for the data at $E=281$, 300 , and especially $330 \mathrm{MeV}$. It is evident that the theoretical calculations using a conventional single-channel DF potential fail to reproduce the experimental data at $281 \sim 330 \mathrm{MeV}$. This shows that behavior not explained by the global potential (visualized by green points) is a nuclear rainbow that is not understood by the traditional concept of rainbow scattering.

Next we show that the disagreement in Fig. 1 is not a drawback of the used global DF potential but a manifestation of the emergence of a new rainbow, which is beyond the optical potential model description of the nuclear rainbow in the Luneburg lens picture [6]. We calculate the ${ }^{16} \mathrm{O}+{ }^{12} \mathrm{C}$ scattering with the EDF potential using $\mathrm{CC}$ method, which takes into account coupling to the excited $2^{+}$and $3^{-}$states of the deformed ${ }^{12} \mathrm{C}$ at the excitation energy 4.44 and $9.64 \mathrm{MeV}$, respectively. The strength of the imaginary potential was slightly reduced since the channel coupling is explicitly introduced, and the radius parameter and the diffuseness parameter were fixed at around 5.6 and $0.75 \mathrm{fm}$, respectively (Table I). In Fig. 2, calculated angular distributions are displayed in comparison with the experimental data. We see that the agreement between the calculations (blue solid lines) and the experimental data is considerably improved compared with Fig. 1. (As discussed later, the larger oscillations than the data at the large angles are reduced by including additional coupling to the excited states of ${ }^{16} \mathrm{O}$ explicitly as displayed by the green thick solid line and the fit to the data is further improved.) The calculation reproduces the new Airy minimum of the nuclear rainbow in the experimental data at $281 \sim 330 \mathrm{MeV}$ well. The rainbow angle, $\theta_{R}$, where the fall-off of the cross sections of the dark side of the rainbow scattering starts is shifted backward in accordance with the appearance of a new Airy minimum. Since the new minimum, $A 1^{(S)}$, at around $\theta=60 \sim 70^{\circ}$ just before the bump is caused by the farside scattering (dashed lines), it is clear that the new Airy minimum is produced by the refractive scattering with channel coupling. Even at $E_{L}=200,230$, and $260 \mathrm{MeV}$, the agreement of the calculated angular distribution with the experimental data is considerably improved in the regions $\theta>100^{\circ}, \theta=80^{\circ} \sim 100^{\circ}$, and $\theta=70^{\circ} \sim 90^{\circ}$, respectively.

As shown at $E_{L}=281$ and $330 \mathrm{MeV}$ in Fig. 2, in creating a new Airy minimum, $A 1^{(S)}$, at the larger angles the coupling to the $2^{+}$state is dominant, while the effect of the $3^{-}$state is negligible and can be simply incorporated into the imaginary 
TABLE I. The volume integral per nucleon pair $J_{V}$ of the the ground-state diagonal potential and the imaginary potential parameters used in the single-channel double-folding calculations in Fig. 1 and coupled channels calculations with EDF in Fig. 2.

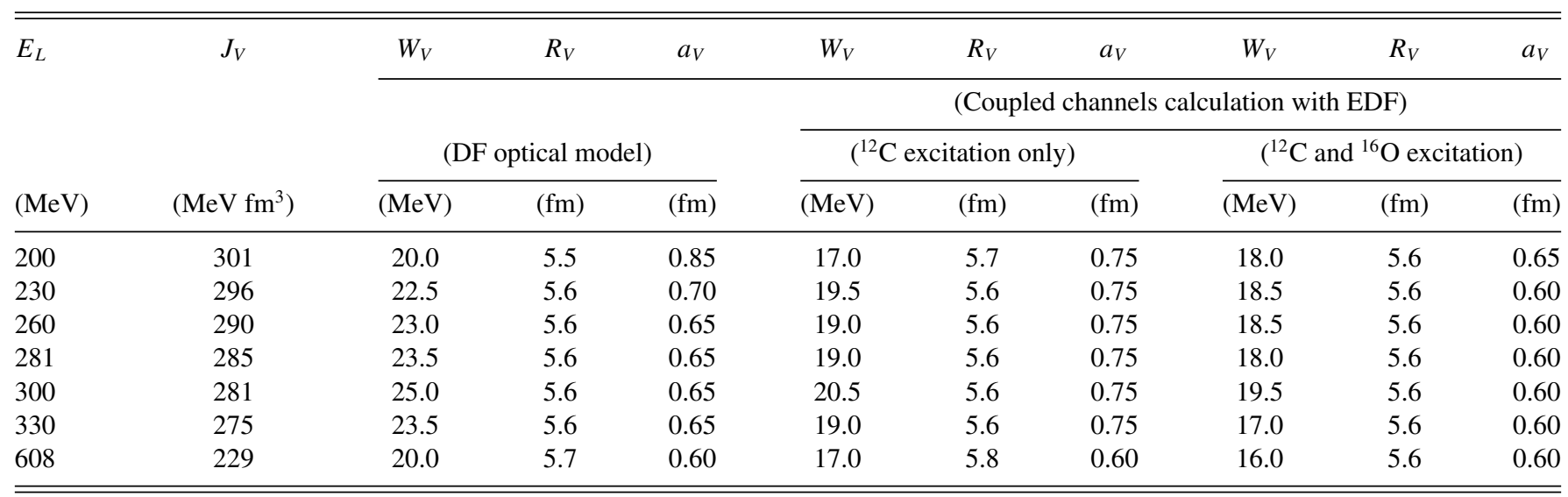

potential. Refraction via coupling to the $2^{+}$state plays the role of a dynamical secondary lens, which is completely different from the ordinary static lens of the nuclear potential interpreted as a Luneburg lens [6]. The effect of the dynamical lens is not possible to incorporate into the DF optical model potential as a normalization constant which is often used in the conventional DF model approach [28]. For this reason the global conventional DF optical model potential failed to reproduce the Airy structure in the $281 \sim 330 \mathrm{MeV}$ data in Fig. 1 .

By investigating the deflection function we show that this new Airy minimum, $A 1^{(S)}$, of the nuclear rainbow in the range $281 \sim 330 \mathrm{MeV}$ is due to a secondary nuclear rainbow, which is dynamically generated in the classically forbidden angular region, i.e., in the dark side of the ordinary "primary" nuclear rainbow. In Fig. 3 the deflection functions determined from the phase shifts calculated in the $\mathrm{CC}$ method and in the conventional DF optical model without imaginary potential are displayed at $330 \mathrm{MeV}$. In the conventional DF optical model calculation we see a single minimum at the orbital angular momentum $L_{R}=27$ with the rainbow angle $\theta_{R}=63^{\circ}$ consistent with the textbook fact that the nuclear rainbow is caused by a refraction in the nuclear attractive potential [29]. On the other hand, in the CC calculation the deflection function is drastically changed to have more than two extrema, which means the existence of more than two nuclear rainbows in elastic scattering by a new mechanism. The investigation of the $S$ matrix shows that the coupling to the $2^{+}$state is very strong for the relevant $L=20 \sim 35$ region, especially at the minima. The two extrema at $L_{R 0}=31$ with $\theta_{R 0}=18^{\circ}$ and at $L_{R 1}=34$ with the rainbow angle $\theta_{R 1}=43^{\circ}$ are located at forward angles, while the minimum with the largest rainbow angle $\theta_{R 2}=95^{\circ}$ appears at $L_{R 2}=26$. In contrast to the meteorological rainbow, the bright sides of the two rainbows where the Airy maximum and minimum appear with $\theta_{R 1}$ ("primary" nuclear rainbow) and $\theta_{R 2}$ (secondary nuclear rainbow) stand on the same side of the nuclear rainbow. Therefore, the dark side of the "primary" nuclear rainbow where the cross sections fall toward large angles in the angular distribution is masked by the bright side of the secondary nuclear rainbow and difficult to observe. The experimental Airy minimum at the large angles, $A 1^{(S)}$, and the fall-off are due to this secondary nuclear rainbow and it is reasonable that the conventional global DF optical model with a single deflection minimum, which creates an Airy minimum, $A 1^{(P)}$, of the "primary" nuclear rainbow due to the nuclear potential, cannot reproduce the Airy minimum of the secondary nuclear rainbow at $281 \sim 300 \mathrm{MeV}$. The precursor of the secondary nuclear rainbow can already be seen in the poor agreement of the calculated angular distributions in the conventional DF optical model at $260 \mathrm{MeV}$ in Fig. 1. It is startling that a secondary nuclear rainbow appears for the nuclear system where the active mechanism is only refractive. This has not been anticipated from the meteorological secondary rainbow for which reflection is involved [1-3,7,29]. A dynamical second lens is only possible in a quantum system such as the ${ }^{16} \mathrm{O}+{ }^{12} \mathrm{C}$ scattering as demonstrated in this paper. The traditional view that the rainbow is generated by static refraction and reflection of classical concept is changed in nuclear context.

In Fig. 4 calculated results without and with the imaginary potentials are compared. We can confirm that the Airy minimum $A 1^{(S)}$, which appears also in the absence of the imaginary potential, is entirely due to the real potential and is created by coupling to the $2^{+}$state of ${ }^{12} \mathrm{C}$.

We investigate the effects of the channels coupling to the excited states of ${ }^{16} \mathrm{O}$ on the creation of the Airy minimum $A 1^{(S)}$. In Fig. 2 the angular distributions calculated including the channels coupling of the ground state to the excited states, $3^{-}(6.13 \mathrm{MeV})$ and $2^{+}(6.92 \mathrm{MeV})$, of ${ }^{16} \mathrm{O}$ in addition to the excited states of ${ }^{12} \mathrm{C}$ are displayed by the green thick solid line. Potential parameters used are given in Table I. We see that the large oscillations at large angles seen in the blue lines are suppressed and the agreement with the experimental data is improved further especially at the large angles around and beyond the Airy minimum. This coupling tends to obscure the deep Airy minimum created by coupling to the $2^{+}$state of ${ }^{12} \mathrm{C}$ as an imaginary potential generally does. These results show that the Airy minimum $A 1^{(S)}$ is due to the $2^{+}$state of ${ }^{12} \mathrm{C}$. Our $\mathrm{CC}$ calculations using the imaginary potentials interpolated from $E_{L}=330$ and $608 \mathrm{MeV}$ predict the persistency of the Airy minimum of the nuclear rainbow at the higher energies between $E_{L}=350$ and $500 \mathrm{MeV}$. Especially at around 


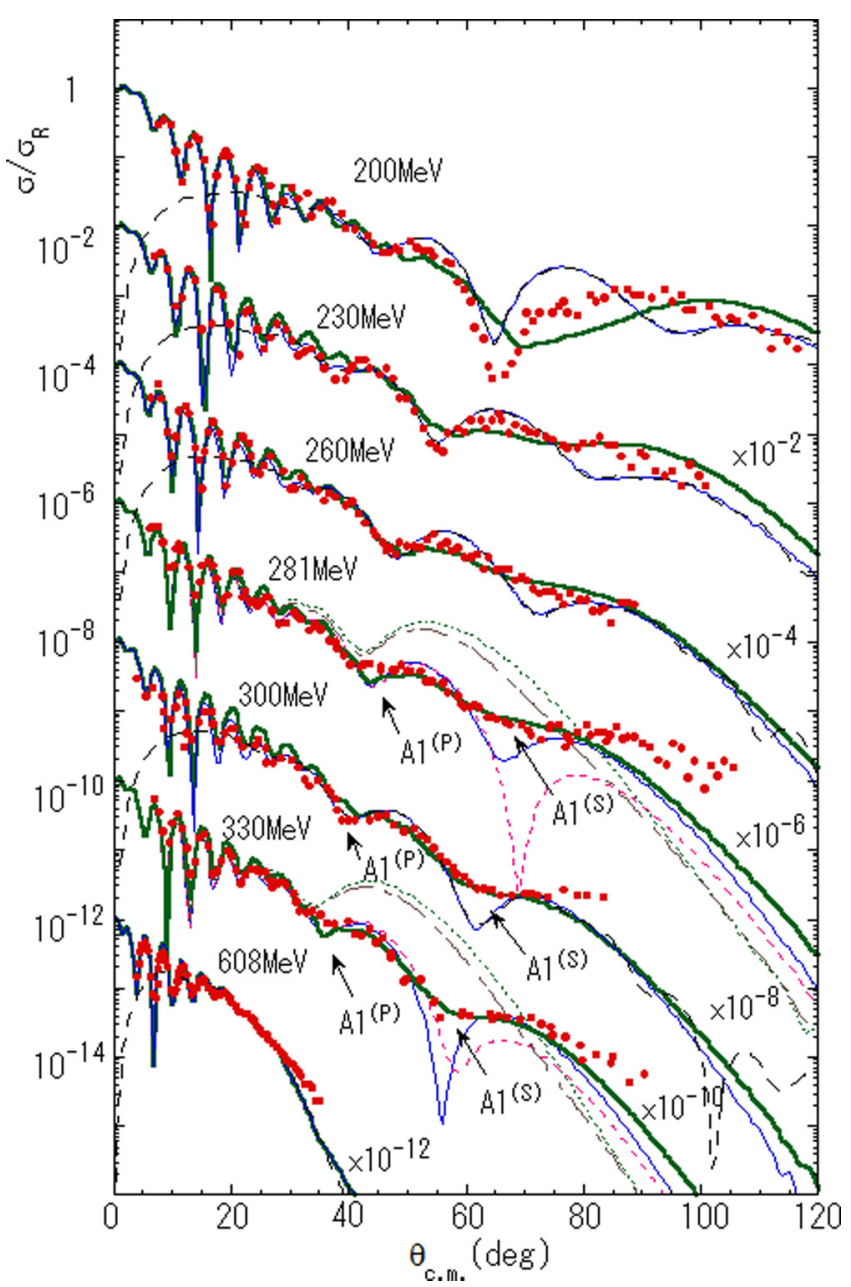

FIG. 2. (Color online) Same as described in the legend of Fig. 1 except for the CC calculations. The calculated cross sections with coupling to the $2^{+}$and $3^{-}$states of ${ }^{12} \mathrm{C}$ (blue solid line) and the farside components (long dashed line) are displayed in comparison with the experimental data (points) [15,25-27]. The calculations with coupling to the $2^{+}$state only (pink dashed line), $3^{-}$state only (dashed dotted line), and no coupling (dotted line) are shown for $E_{L}=281$ and $330 \mathrm{MeV}$. The Airy minimum of the "primary" nuclear rainbow, $A 1^{(P)}$, and that of the secondary nuclear rainbow, $A 1^{(S)}$, are indicated. The $\mathrm{CC}$ calculations including coupling of the ground state to the $3^{-}$ and $2^{+}$states of ${ }^{16} \mathrm{O}$ in addition to coupling to the $2^{+}$and $3^{-}$states of ${ }^{12} \mathrm{C}$ are displayed by the green thick solid line (see text).

$E_{L}=400 \mathrm{MeV}$ a clear deep Airy minimum, which is hardly obscured by the coupling to the excited states of ${ }^{16} \mathrm{O}$, is predicted. The experimental observation of the Airy minimum at these energies is expected.

Finally, we will make a brief discussion on the contribution of the transfer reaction channels. As for the elastic transfer of $\alpha$ particles [30], we see in the recent coupled reaction channel calculations of Ref. [31] that the exchange of the $\alpha$ particle (elastic transfer) is three orders of magnitude smaller than the elastic scattering cross sections. Although a complete description of the data may require both inelastic and exchange couplings, as for the one nucleon exchange effect, which is suggested to prevail over other transfer reactions to affect

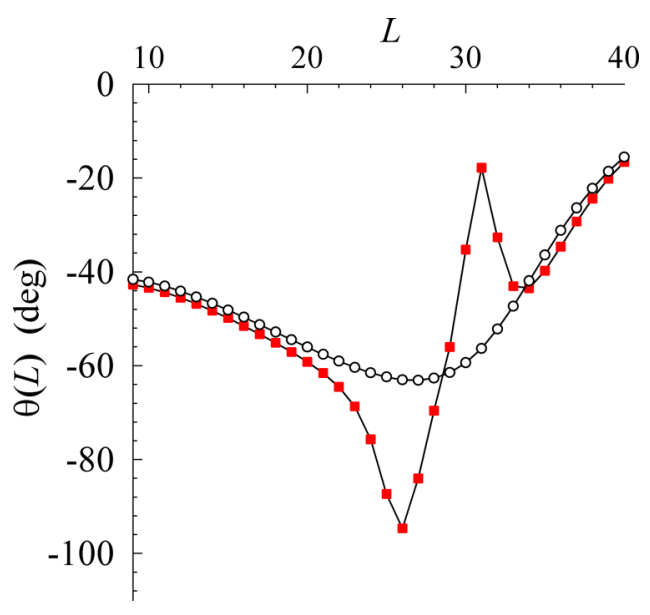

FIG. 3. (Color online) The deflection functions calculated from the real DF optical model (circle) and the CC method (square) with the EDF potential at $E_{L}=330 \mathrm{MeV}$ are compared. The line is to guide the eye.

the Airy minimum $A 1^{(S)}$ for the $230 \mathrm{MeV}$ data in Ref. [31], our calculations take into account it by using the effective interaction DDM3Y, in which the knock-on exchange effect is incorporated [24,28].

To summarize, we have shown for the first time the evidence for the existence of a secondary bow in the Newton's zero-order nuclear rainbow by analyzing ${ }^{16} \mathrm{O}+{ }^{12} \mathrm{C}$ scattering using a coupled channel method with an extended double folding (EDF) potential derived from the microscopic realistic wave functions for ${ }^{12} \mathrm{C}$ and ${ }^{16} \mathrm{O}$. The secondary nuclear rainbow appears dynamically in the classically forbidden angular region of the dark side of the ordinary "primary" nuclear rainbow due to the creation of a new rainbow angle caused by strong coupling to the $2^{+}$state of ${ }^{12} \mathrm{C}$. This plays the

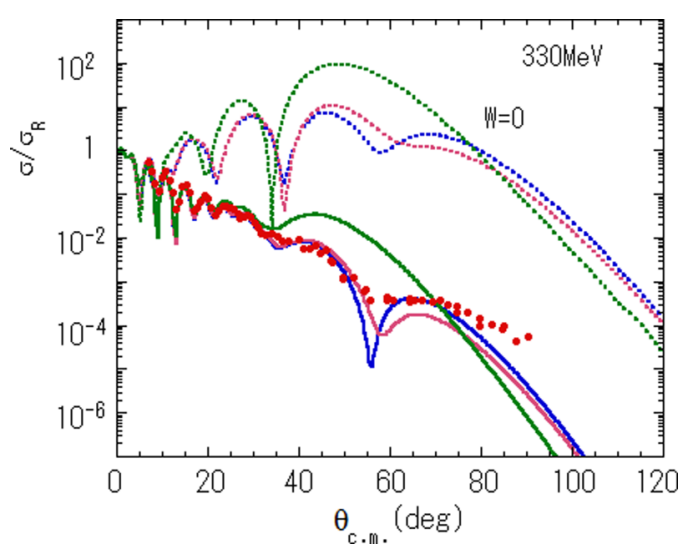

FIG. 4. (Color online) Angular distributions in ${ }^{16} \mathrm{O}+{ }^{12} \mathrm{C}$ scattering at $E_{L}=330 \mathrm{MeV}$ calculated in the single channel (green solid line) and CC method (blue and pink solid line) with the imaginary potential in Table I are compared with those calculated by switching off the imaginary potential (dotted line) and the experimental data (points) [26]. The blue and pink lines represent the $\mathrm{CC}$ results with coupling to the $2^{+}$and $3^{-}$states of ${ }^{12} \mathrm{C}$ and those with coupling to the $2^{+}$state of ${ }^{12} \mathrm{C}$ only, respectively. 
role of a second lens in addition to the static lens caused by the nuclear potential. The traditional view that the rainbow is understood by the classical concept of refraction and reflection is changed in nuclear context. The discovery of a secondary rainbow solves the dilemma concerning the failure of the global potential for the ${ }^{16} \mathrm{O}+{ }^{12} \mathrm{C}$ system. It is desired to observe a secondary rainbow in other systems.
One of the authors (S.O.) thanks the Yukawa Institute for Theoretical Physics for the hospitality extended during a stay in February 2013. Part of this work was supported by the Grant-in-Aid for the Global COE Program "The Next Generation of Physics, Spun from Universality and Emergence" from the Ministry of Education, Culture, Sports, Science, and Technology (MEXT) of Japan.
[1] I. Newton, Opticks or a Treatise of the Reflexions, Refractions, Inflexions, and Colours of Light (Dover publications, New York, 1952).

[2] H. M. Nussenzveig, Sci. Am. 236, 116 (1977).

[3] J. A. Adam, Phys. Rep. 356, 229 (2002).

[4] C. F. Bohren and A. B. Fraser, Am. J. Phys. 59, 325 (1991).

[5] D. A. Goldberg and S. M. Smith, Phys. Rev. Lett. 29, 500 (1972); D. A. Goldberg, S. M. Smith, and G. F. Burdzik, Phys. Rev. C 10, 1362 (1974).

[6] F. Michel, G. Reidemeister, and S. Ohkubo, Phys. Rev. Lett. 89, 152701 (2002).

[7] H. M. Nussenzveig, J. Math. Phys. 10, 82 (1969); 10, 125 (1969).

[8] D. T. Khoa, W. von Oertzen, H. G. Bohlen, and S. Ohkubo, J. Phys. G 34, R111 (2007).

[9] R. Anni, Phys. Rev. C 63, 031601(R) (2001).

[10] F. Michel, S. Ohkubo, and G. Reidemeister, Prog. Theor. Phys. Suppl. 132, 7 (1998).

[11] S. Ohkubo, T. Yamaya, and P. E. Hodgson, in Nucleon-Hadron Many-Body Systems, edited by H. Ejiri and H. Toki (Oxford University Press, Oxford, 1999), p. 150.

[12] S. Ohkubo and K. Yamashita, Phys. Rev. C 66, 021301(R) (2002).

[13] A. A. Ogloblin et al., Phys. Rev. C 57, 1797 (1998).
[14] M. P. Nicoli et al., Phys. Rev. C 61, 034609 (2000).

[15] A. A. Ogloblin et al., Phys. Rev. C 62, 044601 (2000).

[16] S. Szilner et al., Phys. Rev. C 64, 064614 (2001).

[17] D. T. Khoa, W. von Oertzen, and H. G. Bohlen, Phys. Rev. C 49, 1652 (1994).

[18] S. Ohkubo and K. Yamashita, Phys. Lett. B 578, 304 (2004).

[19] A. A. Ogloblin et al., Phys. At. Nucl. 66, 1478 (2003).

[20] S. Okabe, Tours Symposium on Nuclear Physics II, edited by H. Utsunomiya et al. (World Scientific, Singapore, 1995), p. 112.

[21] Y. Suzuki, Prog. Theor. Phys. 55, 1751 (1976); 56, 111 (1976).

[22] Y. Hirabayashi and S. Ohkubo, Phys. Rev. C 88, 014314 (2013).

[23] M. Kamimura, Nucl. Phys. A 351, 456 (1981).

[24] A. M. Kobos et al., Nucl. Phys. A 384, 65 (1982); 425, 205 (1984).

[25] M. E. Brandan et al., Nucl. Phys. A 688, 659 (2001).

[26] A. S. Dem'yanova et al., IAEA Database exfor, http://wwwnds.iaea.org/exfor/.

[27] M. E. Brandan et al., Phys. Rev. C 34, 1484 (1986).

[28] M. E. Brandan and G. R. Satchler, Phys. Rep. 285, 143 (1997).

[29] R. G. Newton, Scattering Theory of Waves and Particles (McGraw-Hill Book Company, New York, 1966).

[30] S. Szilner et al., Eur. Phys. J. A 13, 273 (2002).

[31] A. T. Rudchik et al., Eur. Phys. J. A 44, 221 (2010). 